\title{
Deep Learning Stereo Matching Algorithm using Siamese Network
}

\author{
Masoud Samadi $^{1 *}$, Mohd Fauzi Othman ${ }^{1}$ and Akira Taguchi ${ }^{2}$ \\ ${ }^{1}$ Malaysia-Japan International Institute of Technology, Universiti Teknologi Malaysia, 54100, Kuala Lumpur, Malaysia \\ ${ }^{2}$ Tokyo City University, Tokyo 158-8557, Japan
}

*Corresponding author: mdfauzi@utm.my

\begin{abstract}
Autonomous vehicle has become a very hot topic for researchers in recent years. One of the important sensors used in these vehicles is Stereo Cameras/Vision. Stereo vision systems are used to estimate the depth from the two cameras installed on robots or vehicles. This method can deliver the 3D position of all objects captured in the scene at a lower cost and higher density compared to LIDAR. Recently, neural net-works are vastly investigated and used in image processing problems and deep learning networks which has surpassed traditional computer vision methods specially in object recognition. In this paper, we propose to use a GPU with a new Siamese deep learning method to speed up the stereo matching algorithm. In this work, we use a high end Nvidia DGX workstation to train and test our algorithm and compare the results with normal GPUs and CPUs. Based on numerical evaluation, the Nvidia DGX can train a neural network with higher input image resolution approximately 8 times faster than a normal GPU and 40 times faster than a Core i7 8 Cores CPU. Since it has the ability to train on a higher resolution the network can be trained in more iteration and results in higher accuracy.
\end{abstract}

Keywords: LIDAR, Stereo Vision, Siamese Deep Neural Network, GPU.

Article History: received 13 November 2019; accepted 5 December 2019; published 18 December 2019.

\section{INTRODUCTION}

Autonomous robots and vehicles which need to operate without intervention of any human operators, need to acquire the general knowledge about their surroundings. To obtain this information they use several different sensors. Each sensor is responsible in providing some part of the required data.

In autonomous vehicle industries, cameras are responsible for object detection and recognition, while the depth estimation is done by LIDARs. LIDARs are responsible to detect the distance between the car and other objects in the environment. They are very accurate, but their result is not dense, and they are costly. To reduce the cost of autonomous robots and vehicles, researchers have tried to use cameras as the main depth estimation sensor [1]. Hence, stereo vision systems are invented. Stereo vision uses two cameras which are horizontally aligned for understanding the depth and distance (Figure 1).

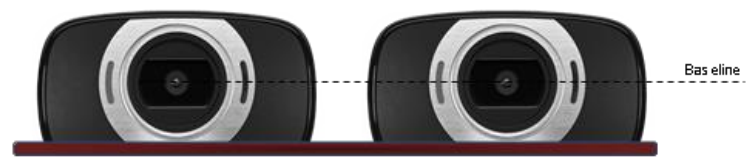

Figure 1. Stereo Vision Cameras

This method has a number of advantages compared to other methods in obtaining 3D data from the environment. Conventional methods contain their own defects and difficulties [2]. Using a pair of cameras for image capturing brings the problem of correspondence between the images obtained.

In general, matching the corresponding points captured by a pair of cameras is very difficult due to the similarities that exist in the images. By employing the stereo matching techniques, the robot can estimate the 3D position of any object in the visibility of its cameras by calculating the stereo disparity for that object. The stereo disparity is the difference between the locations of an object in the two images captured by the stereo cameras and is the result of the stereo matching function [3].

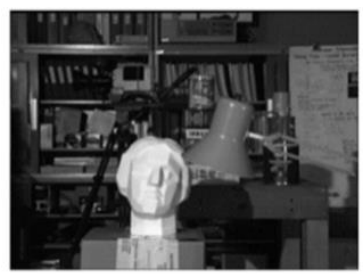

Left Image

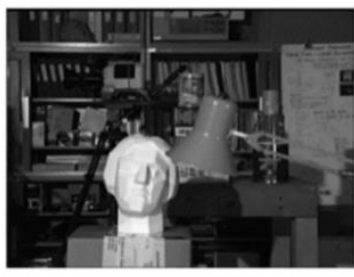

Right Image

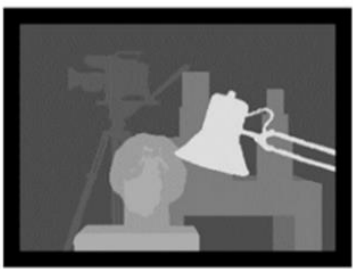

Result: Disparity Map

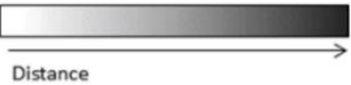

Figure 2. Disparity Map Sample

To realize the $3 \mathrm{D}$ position of all the points in a pair of images from the stereo cameras, the dense disparity map must be computed. Figure 2, at the bottom, shows a 
disparity map that is calculated from the left and right images that are illustrated at the top of the figure. The lighter colors show closer locations to the cameras. It is necessary to mention that the results in Figure 2 constitute a manually made disparity map, which is regularly used by researchers for the purpose of the evaluation of their algorithms.

Stereo matching has been intensively investigated for several decades [19]. Current stereo matching algorithms face problems in repetitive patterns, thin structures, reflective surfaces and textureless areas. Some stereo matching algorithms try to reduce these failures with gradient based regularization or pooling [4, 5]. However, this often requires a compromise between detecting detailed structures and smoothing surfaces.

On the other hand, deep learning models have been successful in learning powerful representations directly from the raw data in object classification [6], detection [7] and semantic segmentation $[8,9]$.

Deep Learning networks are distinguished from the neural net-works by their depth; that is, the number of node layers through which data passes in a multistep process of pattern recognition. Deep learning networks perform automatic feature extraction with-out human intervention, unlike most traditional machine-learning algorithms.

In Deep Learning a Convolutional Neural Network (CNN) is a type of feedforward neural network in which the connectivity pattern between its neurons is inspired by the organization of the animal visual cortex. These networks use a special architecture which is particularly well-adapted to classify images. Using this architecture makes convolutional networks train faster. This, in turn, help to train deep, many-layer networks, which are very good at classifying images. CNNs take advantage of the fact that the input consists of images and they constrain the architecture in a more sensible way. Unlike a regular Neural Network, the layers of a convolutional network have neurons arranged in 3 dimensions, width, height, depth. A typical Convolutional Neural Network architecture is displayed in Figure 3.

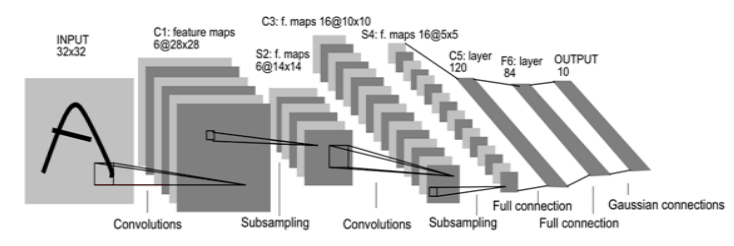

Figure 3. Convolutional Neural Network

\section{LITERATURE}

In recent years, $\mathrm{CNN}$ has been used to solve various problems in stereo matching. Zbontar and LeCun [10] trained a $\mathrm{CNN}$ to compute the matching cost between two 9x9 image patches, which is followed by several postprocessing steps, including crossbased cost aggregation, semi-global matching, left-right constancy check, subpixel enhancement, median filtering and bilateral filtering. This architecture needs multiple forward passes to calculate matching cost at all possible disparities. Therefore, this method is computationally expensive. A multi-scale embedding model from Chen et al. [11] also provides good local matching scores. Another noteworthy example the DeepStereo work of Flynn et al. [12], which learns a cost volume combined with a separate conditional color model to predict novel viewpoints in a multi-view stereo setting.

Park and Lee [13] introduced a pixelwise pyramid pooling scheme to enlarge the receptive field during the comparison of two input patches.

Mayer et al. created a large synthetic dataset to train a network for disparity estimation (as well as optical flow) [14], which leaded to improving the state-of-the-art techniques. As one variant of the network, a 1-D correlation was proposed along the disparity line which is a multiplicative ap-proximation to the stereo cost volume. This is an encoder-decoder architecture for disparity regression. The matching cost calculation is seamlessly integrated to the encoder part. The disparity is directly regressed in a forward pass. Kendall et al. [15] used 3-D convolutions upon the matching costs to incorporate contextual information and introduced a differentiable "softargmin" operation to regress the disparity.

The KITTI dataset $[16,17]$ is a large dataset collected from a moving vehicle with LIDAR ground truth. These datasets first motivated improved hand-engineered techniques for all components of stereo, from which we mentioned a few notable examples.

All of the mentioned method employed $\mathrm{CNN}$ to generate a disparity map in a supervised manner. The KITTI dataset does not provide enough image to train a deep neural network, so the data augmentation technique is used to increase the number of training images for the neural network.

In this work we propose $\mathrm{CNN}$ with residual bocks where we trained and tested it on different platforms. The performance of the Nvidia DGX, Nvidia 1060 GTX and CPU are then reported based the findings.

\section{METHODOLOGY}

In this research we use a Siamese neural network to estimate the depth. Throughout this paper we assume that the image pairs are rectified, thus the horizontal image axis and epipolar lines are aligned. To estimate the depth, we use a Siamese architecture, where each branch processes the left or right image respectively.

Our goal is to minimize the discrepancy between the estimated disparity and ground truth disparity maps, in this case LIDAR images from KITTI dataset.

The first step in any image processing task is a step called feature extraction. Features are small, interesting, descriptive or informative patches in images. In traditional image processing method, this step was one of the most difficult and crucial part of the algorithm, where feature extraction is done manually to extract the best possible description in the image to be used in the next step of the algorithm. In deep learning method, the machine is told to learn what to look for with respect to each specific class of object. It works out for most descriptive and salient features for each object. In other words, neural networks are told to discover the underlying patterns in classes of images, by themselves. Therefore, with deep learning, there is no need to manually decide which traditional computer vision technique to use to describe your features. The machine works this all out itself. Additionally, this approach promises to reduce much of the engineering design complexity. The flowchart of the proposed deep 
learning algorithm is displayed in Figure 4.

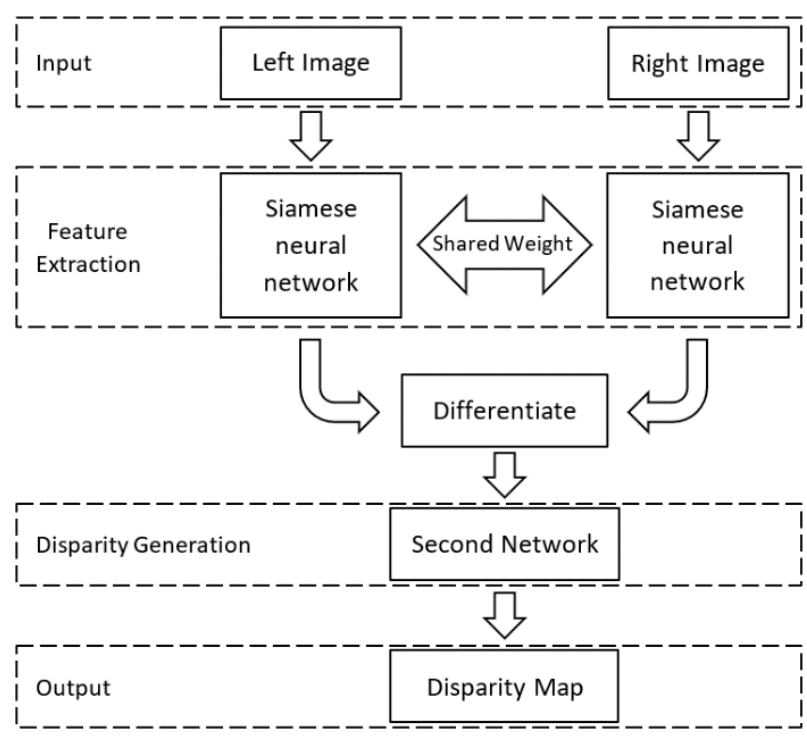

Figure 4. Flowchart of the Proposed Deep Learning Algorithm

In the proposed method, the feature extraction is done by the aforementioned Siamese network. To elaborate in detail, each branch takes an image as input, and passes it through a set of layers, each consisting of a spatial convolution with a small filter-size, followed by the activation function. The first convolutional layer uses a $7 \times 7$ kernel followed by a 3x3 kernel. In this research, we use a rectified linear unit (ReLU) as the activation function. Each even layer is followed by maxpooling with stride 2 , to reduce the image size, or as the researchers call it, downsizing. After 7 layers of $3 \times 3$ convolutions, the downsized feature goes through a series of deconvolution layers with stride 2 to upsample again, while using a residual connection, they are concatenated with the respective conventional layer to increase the feature extraction details.

One of the main problems of a Deep Neural Network is how to propagate the error all the way to the first layer. For a deep network, the gradients keep getting smaller until it has no effect on the net-work weights. Residual connection was designed to overcome such problem (Figure 5), by defining a block with an identity path. During back propagation the gradients have a path that does not affect its magnitude. The network needs to learn residual mapping.
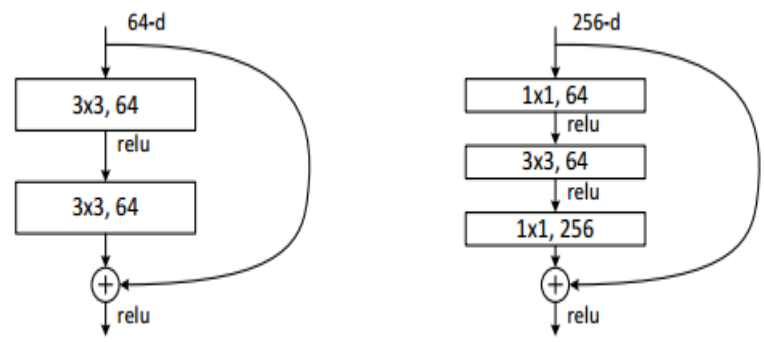

Figure 5. Residual connection Sample

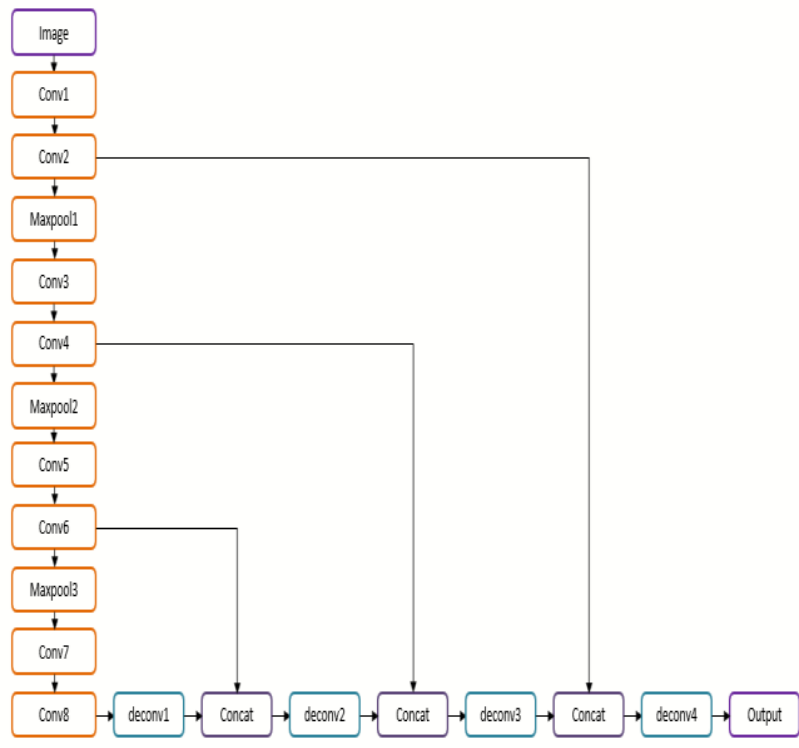

Figure 6. The detailed architecture of each Siamese branch

The result of the two networks are the high descriptive features from left and right images. These features are treated like normal images to generate the disparity map. Inspired by a traditional SAD (Sum of Absolute Differences) method, the two outputs are subtracted, and the result goes through a second network which is used to generated disparity map [18]. Using a second network as the disparity generative method increases the accuracy of the output noticeably.

In the loss function, we would like to minimize the discrepancy between the ground truth and generated disparity map. It can be done by forming a loss, by simply computing the L1 distance between the images and their gradients

$L\left(d_{l}, d^{\prime}{ }_{l}\right)=\frac{1}{N} \sum \lambda_{1}\left|d_{l}-d^{\prime}{ }_{l}\right|+\lambda_{2}\left|\nabla \mathrm{d}_{l}-\nabla \mathrm{d}^{\prime}{ }_{l}\right|$

where $\mathrm{N}$ denotes the total number of pixels and $d_{l}$ is the ground truth and $d^{\prime}{ }_{l}$ is the generated disparity map, $\nabla$ is image gradient. $\lambda_{1}$ and $\lambda_{2}$ are used as coefficient to balance the absoluter difference and the gradient difference.

\section{RESULTS AND ANALYSIS}

In this section the results of the evaluation of the proposed model on the KITTI dataset is reported and the speed comparison between these different datasets is tabulated in Table 1. The network is developed using TensorFlow library [20] and it trained for 20000 epochs of size 50. The network could achieve the error rate of 2.41 in 5 pixel error rate evaluation method which is slightly better than the previous models with error rate of 2.53 . The result and LIDAR ground truth are shown in Figure $6 \& 7$

Please note that the network was trained with a larger size on Nvidia DGX workstation, because the other platform could not support the bigger image size. 

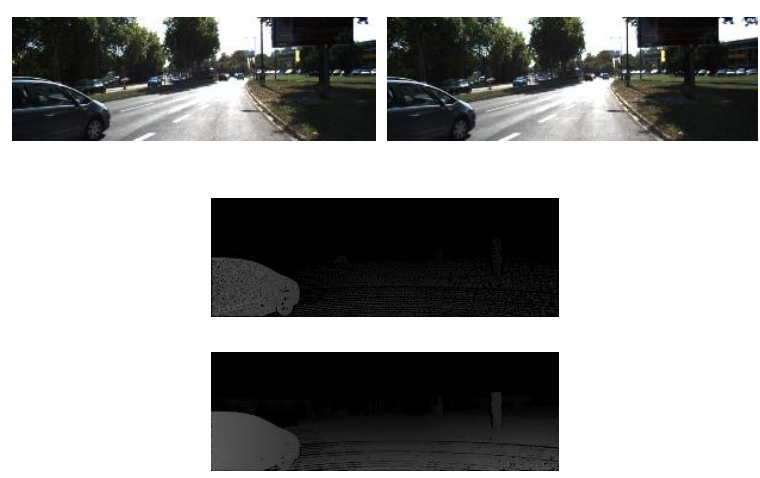

Figure 7. Up: The left camera image, the right camera image The LIDAR (ground Truth) Predicted Disparity map by the proposed method

Table 1. Speed Comparison between different platform

\begin{tabular}{lll}
\hline Platform & $\begin{array}{l}\text { Image } \\
\text { Dimensions }\end{array}$ & Time (ms) \\
\hline Nvidia DGX & $1280 \times 384$ & 0.16 \\
Nvidia 1060 GTX & $960 \times 192$ & 1.30 \\
Intel Core i7 4870 & $960 \times 192$ & 6.40 \\
\hline
\end{tabular}
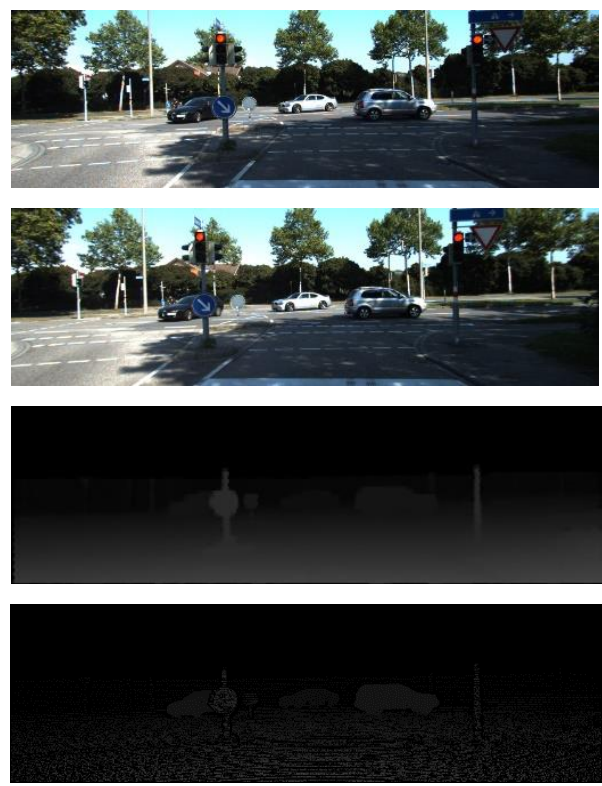

Figure 8. Up: The left camera image, the right camera image The LIDAR (ground Truth) Predicted Disparity map by the proposed method

\section{CONCLUSION}

In this study, we used a Siamese convolutional neural network and trained it using different platforms and reported the results. Based on the observation, the Nvidia DGX can train a neural network with higher input image resolution approximately 8 times faster than Nvidia 1060 GTX GPU and 40 times faster than a Core i7 8 Cores CPU.

\section{ACKNOWLEDGMENT}

We would like to thank the Center for Artificial Intelligence and Robotics (CAIRO), Universiti Teknologi
Malaysia (UTM) and Tokyo City University (TCU) for providing the research facilities.

\section{REFERENCES}

[1] Ben-Tzvi, P. and Xu, X. An embedded feature-based stereo vision system for autonomous mobile robots. Robotic and Sensors Environments (ROSE), 2010 IEEE International Workshop on. 2010. 1 -6.

[2] Wang, L., Liao, M., Gong, M., Yang, R. and Nister, D. High-Quality Real-Time Stereo Using Adaptive Cost Aggregation and Dynamic Programming. Proceedings of the Third International Symposium on 3D Data Processing, Visualization, and Transmission. Washington, DC, USA: IEEE Computer Society.

[3] Samadi M., Othman M.F. (2013) A New Fast and Robust Stereo Matching Algorithm for Robotic Systems. Advances in Intelligent Systems and Computing, vol 209. Springer, Berlin, Heidelberg.

[4] Geiger A., Roser M., Urtasun R. (2011) Efficient Large-Scale Stereo Matching. In: Kimmel R., Klette R., Sugimoto A. (eds) Computer Vision - ACCV 2010. ACCV 2010. Lecture Notes in Computer Science, vol 6492. Springer, Berlin, Heidelberg.

[5] H. Hirschmuller. Accurate and efficient stereo processing by semiglobal matching and mutual information. In 2005 IEEE Computer Society Conference on Computer Vision and Pattern Recognition, volume 2, pages 807-814.

[6] A. Krizhevsky, I. Sutskever, and G. E. Hinton. Imagenet classification with deep convolutional neural networks. In Advances in neural information processing systems, pages 1097-1105, 2012.

[7] R. Girshick, J. Donahue, T. Darrell, and J. Malik. Rich feature hierarchies for accurate object detection and semantic segmentation. In Proceedings of the IEEE conference on computer vision and pattern recognition, pages 580-587, 2014.

[8] Z. Chen, X. Sun, L. Wang, Y. Yu, and C. Huang. A deep visual correspondence embedding model for stereo matching costs. In Proceedings of the IEEE International Conference on Computer Vision, pages 972-980, 2015.

[9] V. Badrinarayanan, A. Kendall, and R. Cipolla. Segnet: A deep convolutional encoder-decoder architecture for image segmentation. DOI :https://doi.org/10.1109/TPAMI.2016.2644615

[10] J. Zbontar and Y. LeCun. Stereo matching by training a convolutional neural network to compare image patches. Journal of Machine Learning Research, 17.

[11] Z. Chen, X. Sun, L. Wang, Y. Yu, and C. Huang. A deep visual correspondence embedding model for stereo matching costs. In Proceedings of the IEEE International Conference on Computer Vision, pages 972-980, 2016.

[12] J. Flynn, I. Neulander, J. Philbin, and N. Snavely. DeepStereo: Learning to Predict New Views from the World's Imagery.

[13] H. Park and K. M. Lee. Look wider to match image patches with convolutional neural networks. IEEE Signal Processing Letters, PP(99):11, 2017.

[14] N. Mayer, E. Ilg, P. H“ausser, P. Fischer, D. Cremers, A. Dosovitskiy, and T. Brox. A Large Dataset to 
Train Convolutional Networks for Disparity, Optical Flow, and Scene Flow Estimation. CoRR, abs/1510.0(2002), 2015.

[15] A. Kendall, H. Martirosyan, S. Dasgupta, P. Henry, R. Kennedy, A. Bachrach, and A. Bry. End-to-end learning of geometry and context for deep stereo regression. In IEEE Conference on Computer Vision and Pattern Recognition.

[16] A. Geiger, P. Lenz and R. Urtasun, "Are we ready for autonomous driving? The KITTI vision benchmark suite," 2012 IEEE Conference on Computer Vision and Pattern Recognition, Providence, RI, 2012, pp. 3354-3361.
[17] M. Menze and A. Geiger. Object scene flow for autonomous vehicles. In Conference on Computer Vision and Pattern Recognition (CVPR).

[18] Samadi M., Othman M.F., Talib M.F. 2016. Fast and Robust Stereo Matching Algorithm For Obstacle Detection In Robotic Vision Systems. 6-13, Jurnal Teknologi

[19] Mahammed M. A., Melhum A. I., Kochery F.A. 2013. Object Distance Measurement by Stereo VISION. International Journal of Science and Applied Information Technology (IJSAIT), Vol.2, No.2, Pages: 05-08

[20] https://www.tensorflow.org/, Tensor Flow opensource deep learning library, Retrieved 15 October 2018. 